\title{
Análisis sobre el impacto social del programa académico de la licenciatura en medicina del Centro Universitario del Sur de la Universidad de Guadalajara, México
}

Analysis of the social impact of the academic program of medical degree from Southern University Center of the University of Guadalajara, Mexico

Donato Vallín González

Universidad de Guadalajara, México

dvallin@cucsur.udg.mx

Francisco Santillán Campos

Centro de Estudios e Investigaciones para el Desarrollo Docente CENID, México

cenidorgmx@gmail.com

\section{Resumen}

Con la idea de conocer el impacto en la sociedad que ha tenido la licenciatura en medicina del Centro Universitario del Sur de la Universidad de Guadalajara ${ }^{2}$, localizado en Ciudad Guzmán, Jalisco, México, se aplicó la metodología de GRANA ${ }^{3}$, que se basa en un sistema decimal de evaluación cualitativa y cuantitativa compuesto por 10 rubros o dimensiones, 100 ítems y 1000 indicadores de segunda y tercera generación. Sus referentes son la $\mathrm{UNESCO}^{4}$, la $\mathrm{OCDE}^{5}$, el Banco Mundial ${ }^{6}$, la declaratoria de Bolonia ${ }^{7}$, entre otros. Se

utiliza una plataforma informática llamada $\operatorname{SIEVAS}^{8}$ que facilita el llenado de la evaluación y permite la interacción entre evaluadores. Las herramientas que posee para facilitar la evaluación son: significados, contextos, referencias bibliográficas, glosario, escala decimal para calificar niveles de calidad, campos para integrar las fortalezas y las debilidades, el plan de mejora permanente, formatos para integrar las estadísticas en retrospectiva y prospectiva, disponibilidad informática para incorporar documentos probatorios y sitios web que ayudan al proceso de evaluación. También cuenta con gráficos que se construyen 
automáticamente conforme se incorpora la evaluación a la plataforma. Existen 10 pasos para realizar todo el proceso de evaluación, iniciando con la evaluación interna, después con la evaluación externa y con la entrega de resultados. La evaluación interna es realizada por un comité de la institución evaluada compuesto por profesores, directivos, estudiantes, egresados y empleadores. Mientras que los evaluadores externos provienen de tres continentes, son especialistas en la disciplina a evaluar y han tenido experiencia en procesos de evaluación en su país o región de origen. La sección de evaluación del rubro uno, denominado "Impacto social de la formación", está conformada por 10 ítems que fueron analizados para la construcción de este artículo.

Palabras clave: calidad, pertinencia, SIEVAS, GRANA, evaluación interna, evaluación externa.

\section{Abstract}

With the idea of knowing the impact on society that has had a degree in medicine from the University Center of Southern University Guadalajara2, located in Ciudad Guzman, Jalisco, Mexico, the methodology GRANA3, which is based on a system it was applied decimal qualitative and quantitative evaluation consisting of 10 items or dimensions, 100 items and 1000 indicators second and third generation. Their references are UNESCO4, OECD5, the Mundial6 Bank, the declaration of Bolonia7, among others. a software platform called SIEVAS8 that facilitates filling of the assessment and allows interaction between evaluators used. Its tools to facilitate evaluation are: meanings, contexts, references, glossary, decimal scale to rate levels of quality, fields to integrate the strengths and weaknesses, the plan of continuous improvement, formats for integrating statistics retrospective and prospective, computer availability to include supporting documents and websites that help the evaluation process. It also features graphics that are built automatically as the platform evaluation is incorporated. There are 10 steps to make the whole evaluation process, starting with internal assessment, after the external evaluation and delivering results. The internal evaluation is conducted by a committee of the evaluated institution composed of teachers, administrators, students, graduates and employers. While external evaluators from three continents, are specialists in the discipline to evaluate and have had experience in evaluation processes in their country or region of origin. The 
evaluation section of the category one called "Social impact of training", consists of 10 items that were analyzed for the construction of this article.

Key words: quality, relevance, SIEVAS, Grana, internal evaluation, external evaluation.

Fecha recepción: Enero 2015 Fecha aceptación: Julio 2015

\section{Introducción}

La Universidad de Guadalajara $^{2}$ es una institución educativa reconocida dentro de las 30 mejores universidades de América Latina ${ }^{9}$, y es la segunda casa de estudios más importante de todo el país. De manera específica, el programa educativo de licenciado en medicina o médico cirujano y partero de la red universitaria está considerado dentro de los 10 mejores por su calidad en la educación a nivel nacional; a nivel, local representa el primer lugar de las escuelas de medicina en el estado por su prestigio, programa y calidad. ${ }^{10}$

El plan de estudios es pertinente en comparación con otros programas nacionales e internacionales, tanto en el nombre de la licenciatura como en los años de estudio, el mapa curricular y el perfil de egreso.

Dentro de las fortalezas del programa educativo encontramos que el diseño del plan de estudios permite una incorporación, desde los primeros semestres, a las áreas clínicas, en el entendido de que la práctica es fundamental en el proceso de aprendizaje del futuro médico cirujano y partero; esto se logra porque la Universidad de Guadalajara cuenta con dos hospitales escuela, además de convenios con hospitales que permiten la incorporación temprana a la práctica médica, ayudando a fortalecer el desarrollo de habilidades y competencias de los estudiantes para su práctica profesional, y contribuyendo así al perfil de egreso del programa académico que contempla una visión local, nacional e internacional. 
Estas competencias que adquieren los estudiantes, gracias al diseño del plan de estudios, han permitido el reconocimiento de otras universidades e instituciones de salud, y esto se ve reflejado en la aceptación de la movilidad estudiantil a nivel nacional e internacional.

El programa de la LMCP de la Universidad de Guadalajara tiene una significativa presencia en el estado de Jalisco, debido a que se imparte en un centro temático y en cuatro centros regionales, lo cual permite el ingreso de estudiantes no solo de origen regional, sino también nacional. El programa de la LMCP ha sido reconocido por la Secretaría de Educación Pública (SEP) ${ }^{11}$ de México como un programa de calidad. El egresado puede obtener el reconocimiento de sus estudios por la Secretaría de Educación Pública (SEP) de México al realizar el examen de certificación de Exámenes Generales para el Egreso de la Licenciatura (EGEL), del Centro Nacional de Evaluación para la Educación Superior, A.C. $(\text { CENEVAL })^{12}$, de acuerdo con el marco jurídico de México.

La Universidad de Guadalajara, a través del programa académico de la LMCP en el Centro Universitario del Sur (CUSur) ${ }^{13}$, permite que la ubiquen entre las nueve Universidades que pertenecen al Padrón de Programas de Licenciatura de Alto Rendimiento AcadémicoEGEL. La publicación de escalas universitarias se ha constituido en un referente importante en la valoración social del desempeño de las instituciones de educación superior. El Examen Nacional para Aspirantes a Residencias Médicas (ENARM) ${ }^{14}$ es un instrumento de medición de conocimientos de medicina general. La tendencia del ingreso de sustentantes de la Universidad de Guadalajara es ligeramente a la alza a través de los años (2009-2014) y ocupa segundo lugar en promedio y número de admitidos en ENARM 2014, si consideramos más de 1000 sustentantes, colocando a la Universidad de Guadalajara en una muy adecuada posición a nivel nacional. Es importante acotar que tanto en el 2014 como en el 2015 el promedio más alto a nivel nacional lo obtuvo un egresado del PA de la LMCP. El programa de la LMCP es relevante académicamente y responde a necesidades locales, regionales, nacionales e internacionales. El programa ha definido un proyecto educativo coherente con el proyecto institucional, en el cual se señalan los objetivos, los lineamientos básicos del currículo, las metas de desarrollo, las políticas y estrategias de planeación y evaluación, y el sistema de aseguramiento de la calidad. El programa es de dominio público. Toda la información anteriormente referida está integrada en el SIEVAS y fue corroborada durante las visitas y entrevistas realizadas al CUSur por los evaluadores 
externos. La evaluación interna y externa del rubro "Impacto social de la formación" se desarrolló bajo los siguientes ítems: 1.1. Coherencia entre la visión, la misión y los objetivos planteados en la génesis de la formación con los resultados actuales; 1.2. Participación de entes internos y externos a la institución en la planeación; 1.3. Pertinencia y competitividad en el ámbito nacional e internacional; 1.4. Reconocimiento de la sociedad a los egresados por su desempeño; 1.5. Competitividad de los egresados ante similares externos; 1.6. Percepción de la comunidad científica, colegios especializados, egresados y empleadores; 1.7. Impacto en la inserción laboral nacional e internacional; 1.8. Percepción de los estudiantes, profesores y personal administrativo; 1.9. Egresados inscritos en el posgrado; y 1.10. Participación de egresados en la mejora de la formación. Fueron diezpasos aplicados para esta evaluación, iniciando con la evaluación interna, siguiendo con la evaluación externa de pares expertos en la disciplina y la entrega de resultados. Se trabajó con la metodología de GRANA, y la plataforma informática del SIEVAS. Los evaluadores expertos pares fueron de tres continentes. ${ }^{15}$

\subsection{Materiales y métodos}

Para realizar este artículo fue necesario analizar los procesos, los procedimientos y los resultados obtenidos en la evaluación al programa académico que se desarrollan a través de la metodología de GRANA. ${ }^{16}$ Este modelo se lleva a cabo de acuerdo con los siguientes momentos:

3.2.1. Conformación del Comité de Evaluación Interna (CEI). Se integra por un equipo de trabajo compuesto por profesores, directivos, estudiantes, egresados y empleadores asociados al programa académico a evaluar.

3.2.2. Asignación de claves al CEI para acceso a la plataforma y sistema de evaluación SIEVAS.

3.2.3. Capacitación en línea y roles del sistema SIEVAS. El sistema SIEVAS cuenta con una plataforma informática que da soporte tecnológico al proceso de evaluación interna, externa y de mejoramiento permanente a los programas (PA) evaluados o a evaluar. Sus servidores se localizan en Phoenix, Arizona, y cuentan con un vasto campo de almacenaje y seguridad para el hospedaje y protección de la información que es colocada durante los procesos de evaluación. Los roles del sistema SIEVAS son: 
1. Administrador del sistema. Tiene acceso a la funcionalidad completa del sistema, además de ser encargado del ingreso de la información correspondiente a la parametrización, la creación de usuarios, programas e instituciones a evaluar.

2. Evaluador interno. Es quien debe dar cuenta de la calidad del programa o institución evaluada; para ello deberá dar respuesta a cada uno de los ítems, identificando fortalezas, debilidades, acciones de mejoramiento y documentos soportes de la información ingresada, y presentar la información estadística en los formatos dispuestos.

3. Evaluador externo. Evaluación hecha por pares externos teniendo como referencia los resultados obtenidos en la evaluación interna.

4. Experto. Consiste en llevar un registro de la experiencia de personas expertas en las diferentes áreas de conocimiento, y que se pueda tener como referencia de acuerdo con los temas de interés, accediendo a un foro que se jerarquiza por áreas y núcleos de conocimientos.

5. Consultor. Es un usuario que podrá ver la información ingresada al sistema, pero solo a manera de consulta, es decir, que no podrá escribir ni modificar la información ingresada por los responsables de las evaluaciones.

6. Sistema decimal de calidad. Para facilitar los procesos de evaluación, GRANA conformó un sistema métrico decimal para la medición de la calidad, denominado sistema VALLIN, que consiste en: 10 rubros-dimensiones, 100 ítems (10 ítems por 1 rubrodimensión), 1000 indicadores de segunda y tercera generación (10 indicadores por ítem). La escala de calidad es decimal 0-10 (donde 0 es calidad nula y 10 es excelente). Son 10 los procesos a realizar y 100 procedimientos a lo largo de la evaluación. En tiempo son 100 días promedio para cada proceso de evaluación. Son 10 tablas o formatos para la elaboración estadística de la evaluación.

7. Modelo de evaluación circular y cíclico. El modelo de evaluación GRANA es actualizado permanentemente bajo un esquema circular y cíclico, como se muestra en la figura 1. Este modelo aplica a su vez en los procesos de mejoramiento permanente (PMP) de las entidades evaluadas de las instituciones de educación, como en los programas académicos. 


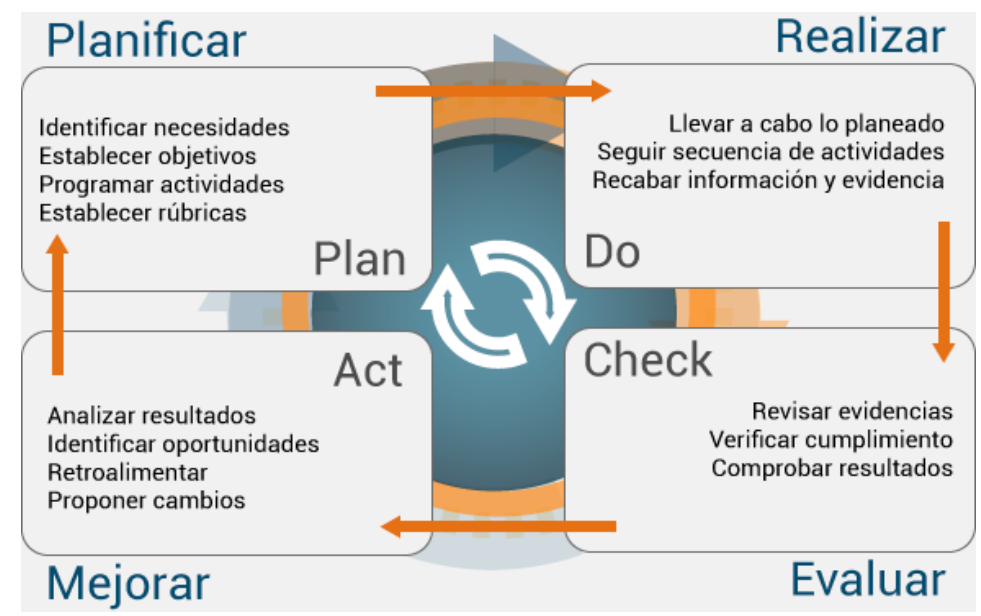

Figura 1. Modelo de evaluación GRANA, cíclico y circular.

3.2.4. Evaluación interna (EI). EI se inicia con la integración y sistematización de la información referida a la unidad objeto de evaluación. El Comité de Evaluación Interna (CEI) integrará al SIEVAS la información de la EI, mediante las observaciones, opiniones y valoraciones generadas a lo largo del proceso. Esta evaluación consiste en que el CEI construya puntual y objetivamente:

a) Integración del FODA del PA.

b) Integración de información estadística del PA.

c) Construcción del plan de mejora permanente (PMP) del PA.

d) Integración de documentos probatorios del FODA y datos estadísticos del PA. Toda esta información es integrada al SIEVAS, como se observa en la figura 2. 


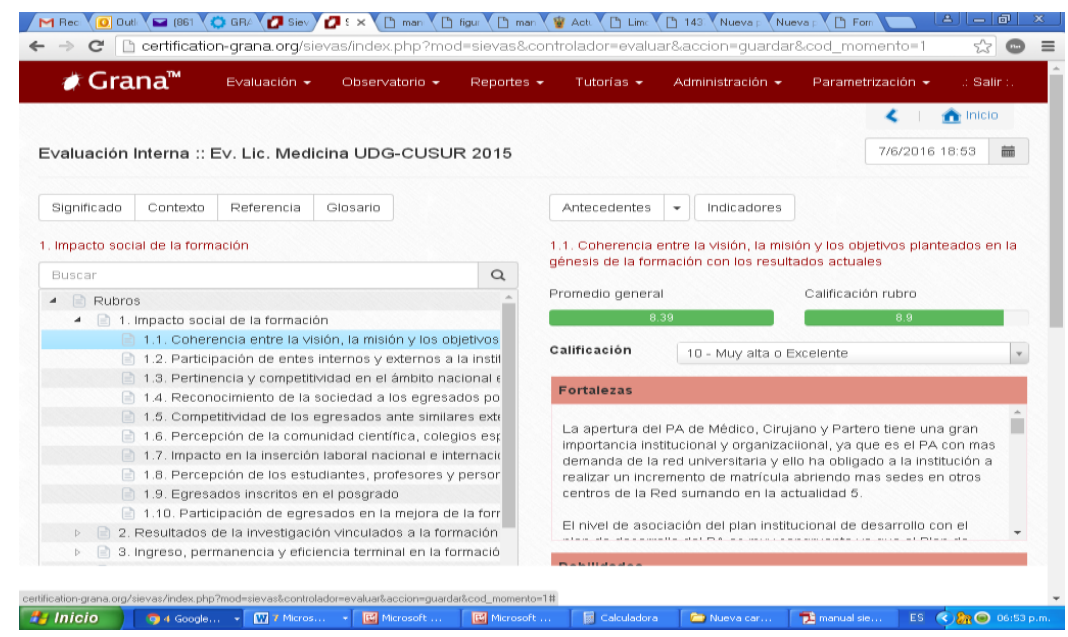

Figura 2. Vista de la plataforma SIEVAS.

3.2.5. Selección de evaluadores externos (EE). Se analizan perfiles requeridos de académicos destacados en la disciplina a evaluar, con conocimiento y experiencia en evaluación académica. Los EE serán de distintos continentes.

3.2.6. Evaluación externa en línea. Mediante la plataforma SIEVAS se llevan a cabo los procesos de evaluación externa; en ella se plasman las coincidencias y diferencias del análisis previo a la visita in situ a la institución evaluada.

3.2.7. Visita a las instalaciones de los evaluadores externos. Para verificar información y realizar entrevistas a los principales involucrados en la evaluación, se realiza una visita de tres días a las instalaciones de la institución evaluada.

3.2.8. Entrega de predictamen a la institución. Al final de la visita es entregado un preinforme a la institución evaluada.

\subsubsection{Reporte-dictamen de resultados de la evaluación y plan de mejora permanente.}

Después de la visita a la institución, y en un plazo no mayor a 30 días, los evaluadores externos entregan el reporte-dictamen sobre el proceso de evaluación. En este reporte se describen las recomendaciones para la mejora permanente del PA evaluado.

3.2.10. Metaevaluación del proceso. El proceso también es evaluado por la institución evaluada para la mejora del modelo y el sistema de SIEVAS de GRANA. 
Sobre el sistema y los criterios de evaluación de GRANA y del SIEVAS. La escala de medición es cualitativa y cuantitativa, y se refiere a niveles de capacidad, competitividad, pertinencia, cobertura e innovación del programa o institución; en ella se establecen los valores de calidad asociados al juicio de valor otorgado a cada ítem y al resultado de calidad obtenido una vez finalizada la evaluación. La escala definida es la siguiente: $0=$ Nulo, 1 = Muy escasa, $2=$ Escasa, 3=Medianamente escasa, $4=$ Poco incipiente, $5=$ Medianamente incipiente, $6=$ Incipiente, 7 = Incipientemente alta, 8=Medianamente alta, 9 $=$ Alta, $10=$ Muy alta o excelente.

El SIEVAS cuenta con gráficos dinámicos que permiten visualizar en línea y en tiempo real el nivel de calidad con que las evaluaciones interna y externa del PA van desarrollándose, como se muestra en la figura 3: el círculo de color rojo al centro representa el nivel bajo en la calidad, el círculo amarillo céntrico representa el nivel medio de calidad y el último círculo de color verde representa el nivel alto de calidad; la sección de color azul representa las fortalezas del PA evaluado, mientras que la zona no cubierta de azul son las oportunidades del PA.

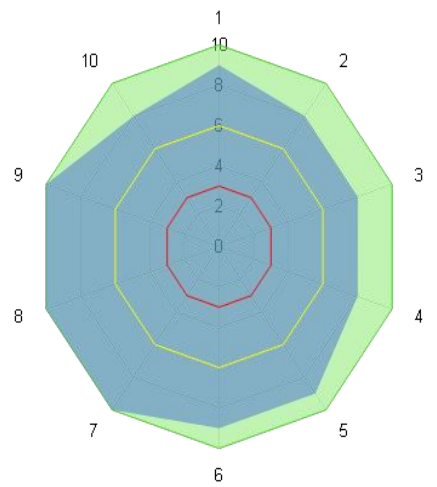

Figura 3. Gráfica de la calidad de GRANA. 


\subsection{Resultados}

\subsubsection{Análisis interno del CEI del programa académico (PA) evaluado.}

\subsubsection{La coherencia entre la visión, la misión y los objetivos planteados en la génesis} de la formación con los resultados actuales.

Fortalezas. La apertura del PA de médico, cirujano y partero tiene una gran importancia institucional y organizacional, ya que es el PA con más demanda de la red universitaria y ello ha obligado a la institución a realizar un incremento de matrícula, y a abrir más sedes en otros centros de la red, que suman en la actualidad 5.

El nivel de asociación del plan institucional de desarrollo con el plan de desarrollo del PA es muy congruente, ya que el Plan de Desarrollo Institucional (PDI) ${ }^{17}$ define las estrategias, objetivos y metas que deben seguir todos los PA; el plan de desarrollo del programa (PDP) proyecta cumplir las estrategias, objetivos y metas institucionales, pero inherentes a la carrera de medicina. Los PDP se modifican y adaptan cuando el PDI se actualiza en la institución, pero consideran los logros obtenidos desde su inicio hasta el momento actual, procurando siempre la constante mejora del programa en los aspectos de gestión, investigación, docencia y vinculación,

Los recursos financieros del presupuesto ordinario de la Universidad garantizan el desarrollo del PA atendiendo diversos programas de extensión, difusión, vinculación, asesoría y tutoría para los estudiantes, profesores, personal administrativo y directivo. Adicionalmente estos recursos permiten promover y desarrollar algunas actividades de internacionalización.

Tanto a nivel del PDI como del PDP se priorizan la calidad, la pertinencia y la prospección, mediante los procesos de planeación y evaluación del PA apoyados en un sistema de gestión de calidad certificado por la Norma ISO 9001-2008.

El PDI considera a la internacionalización como un eje prioritario para el 2030, y a través de él se contempla una serie de objetivos y metas que deben cubrir los PDP en el cumplimiento de sus funciones sustantivas, lo que favorece acciones de proyección a futuro que permitirán que los estudiantes y académicos del PA tengan facilidades para realizar acciones de movilidad internacional.

Con fundamento en los objetivos del eje estratégico de docencia del PDI y considerado en el PDP del PA, recientemente se actualizó y rediseñó curricularmente el PA con el 
propósito de ponerlo a punto con base en el contexto nacional e internacional de las competencias que requerirán los médicos en 2030.

Para la elaboración y evaluación del PDP de medicina fueron considerados diversos sectores de la sociedad, como los colegios y asociaciones médicas, así como directores de hospitales del sector salud y jefes de enseñanza. ${ }^{18}$

El PDI, el Plan de Desarrollo del Centro (PDC) y el PDP contemplan ejes transversales y horizontales de la investigación, la docencia, la extensión y la tutoría como actividades que favorecen e impactan el desarrollo pertinente del PA.

Debilidades. No existe una participación activa de los sectores de la sociedad en la elaboración del plan de desarrollo del PA.

Plan de mejoramiento. Incluir a los sectores de la sociedad vinculados con el área médica, como: colegios de profesionistas, asociaciones médicas, directores de hospitales y jefes de enseñanza en la elaboración del PDP del PA.

Calificación: 10-Muy alta o excelente

\subsubsection{Participación de entes internos y externos a la institución en la planeación}

Fortalezas. La Universidad de Guadalajara desarrolla permanentemente acciones de formación y actualización para el personal que la integra en todos los niveles, con el propósito de reforzar las capacidades que le permitan cumplir de manera eficaz con sus funciones y actividades. Como parte de este proceso, y en el marco del programa institucional de formación y actualización, se diseñó el Diplomado en Gestión Educativa, dirigido a los coordinadores de pregrado de la red universitaria. El Diplomado ofreció a los coordinadores de pregrado elementos de formación y actualización para la gestión pertinente, y brindó herramientas para fortalecer el diseño del plan de gestión de los programas educativos; ofreció elementos formativos que permiten potenciar los aspectos académicos de los programas de pregrado, elevar su calidad y contribuir al cumplimiento de los objetivos y metas institucionales. Además, durante el Diplomado se contó con la intervención de docentes locales, nacionales e internacionales que apoyaron para el cumplimiento de los objetivos.

\section{Debilidades}


La internacionalización de egresados es mínima.

Plan de mejoramiento. Se plantea revisar cada 10 años el currículo, y en esa revisión se propone comparar y equiparar el PA con similares a nivel mundial.

Calificación: 9-Alta

\subsubsection{Pertinencia y competitividad en el ámbito nacional e internacional}

Fortalezas. La Universidad de Guadalajara, en conjunto con los hospitales escuela, realiza y organiza diversos eventos académicos y congresos de talla internacional que permiten la cooperación y competitividad en el ámbito nacional e internacional.

Los egresados del programa académico de médico cirujano y partero de la Universidad de Guadalajara se han destacado por sus competencias profesionales, lo que los ha llevado a ocupar cargos en puestos directivos, como secretarios de salud, jefes de residentes, directores de hospitales, entre otros, e incluso se encuentran ejerciendo la profesión de manera sobresaliente fuera del país.

El programa de médico cirujano y partero se encuentra reconocido por el padrón de alto rendimiento del CENEVAL.

Debilidades. Insuficiente difusión de la Universidad y del programa educativo a nivel internacional. A nivel Latinoamérica existe ya un reconocimiento fundamentado del programa académico, pero falta lograr que ese reconocimiento se consolide en Norteamérica y en otros continentes.

Plan de mejoramiento. Generar estrategias de difusión de la Universidad y del programa educativo a nivel internacional, principalmente en América del Norte y otros continentes.

Calificación: 10-Muy alta o excelente.

\subsubsection{Reconocimiento de la sociedad a los egresados por su desempeño}

Fortalezas. Los egresados del PA de médico cirujano y partero que se imparte en el Centro Universitario del Sur (CUSur) ${ }^{13}$ han tenido muy buena aceptación y reconocimiento en sus actividades debido a su formación integral y a su gran desempeño académico. 
En las evaluaciones nacionales del Examen Nacional de Residencias Médicas (ENARM) ${ }^{14}$ en los últimos 14 años, por los resultados obtenidos, el CUSur se ubica dentro de los primeros 30 lugares, y de manera particular, en el año 2014 alcanzó el lugar $27^{\circ}$, con un promedio de 62.56 puntos.

Adicionalmente, los egresados tienen un alto porcentaje de calificación en el Examen General de Egreso de la Licenciatura (EGEL) que aplica el Centro Nacional de Evaluación para la Educación Superior CENEVAL ${ }^{12}$, donde un número importante de los egresados ha obtenido resultados sobresalientes, la mayoría por arriba de la media nacional.

A nivel del CUSur se han realizado cuatro estudios de seguimiento de egresados en los últimos 7 años, donde se incluye a la carrera de médico, cirujano y partero, solo que en dicho estudio se hicieron muestreos de varios programas educativos donde se muestran resultados que expresan el buen desempeño en las instituciones nacionales de salud.

Tenemos en la actualidad alumnos que se encuentran cursando estudios de posgrado y adiestramientos en instituciones nacionales, en Estados Unidos de América, Brasil, Austria y España.

\section{Debilidades}

No se cuenta con estudios de seguimiento de egresados específicos del programa educativo que considere a $100 \%$ de los egresados.

No se tiene un registro permanente y sistematizado de los reconocimientos obtenidos por los egresados del programa educativo de medicina.

No se cuenta con un plan estratégico para consolidar el reconocimiento social al egresado desde las dimensiones nacional e internacional.

Plan de mejoramiento. Se desarrolla un proyecto de seguimiento de egresados específico del programa educativo de médico, cirujano y partero que incluye a $100 \%$ de los egresados.

Se ha implementado un registro permanente y sistematizado de los reconocimientos que obtienen los egresados del programa educativo de medicina a nivel local, regional, nacional e internacional para alcanzar en el primer año 30\% de los que egresaron del 2001 al 2015. Se han implementado mecanismos para desarrollar un plan estratégico que permitirá consolidar el reconocimiento social al egresado desde las dimensiones nacional e internacional. 


\section{Calificación: 9-Alta}

\subsubsection{Competitividad de los egresados ante similares externos}

Fortalezas. Los resultados del CENEVAL muestran que los egresados del CUSur tienen un desempeño sobresaliente, $70 \%$ obtiene resultados de excelencia, sobresaliente y satisfactorio, lo que ubica a los egresados del PE en el margen de las mejores escuelas de medicina del país.

Uno de los parámetros externos que permite evaluar la competitividad de los egresados del CUSur es el Examen Nacional de Residencias Médicas (ENARM), ${ }^{14}$ al posicionarse el PE dentro de los primeros 30 lugares con resultado promedio de 62.5 puntos.

Debilidades. No se cuenta con una sistematización de la actualización de datos de los egresados, por lo que se pierde contacto con ellos al segundo o tercer año de su egreso.

No se cuenta con un plan sistematizado para consolidar la competitividad local, nacional o internacional del egresado.

Plan de mejoramiento. Realizar un plan estratégico que nos permita conocer el desempeño y reconocimiento de nuestros egresados a nivel nacional e internacional.

Calificación: 8-Medianamente alta.

\subsubsection{Percepción de la comunidad científica, colegios especializados, egresados y empleadores}

Fortalezas. La percepción de la comunidad científica, egresados y empleadores hacia la Universidad de Guadalajara es muy buena, y es producto del alto nivel académico con el que egresan nuestros estudiantes.

Las habilidades que desarrollan gracias al plan de estudios los convierten en profesionales competentes en el ámbito laboral. Esto se refleja, por una parte, en la cantidad de médicos que ingresan a una especialidad médica y/o se integran de forma rápida al campo laboral. El prestigio de la Universidad de Guadalajara y del programa educativo de médico cirujano y partero es respaldado por más de 100 años, y posiciona a la universidad como la segunda 
más importante del país y al programa educativo dentro de los primeros ocho de mejor calidad.

Debilidades. Poca difusión de los logros de los egresados; por otra parte, son insuficientes los programas de extensión y difusión, así como los convenios con empleadores.

Plan de mejoramiento. Crear un programa permanente de extensión y difusión para el programa educativo de la red; gestionar campos laborales para egresados de la red; realizar estudios de opinión y percepción de la propia universidad de Guadalajara, enfocados al programa educativo de médico cirujano y partero

Calificación: 10-Muy alta o excelente.

\subsubsection{Impacto en la inserción laboral nacional e internacional}

\section{Fortalezas}

La inserción al campo laboral por parte de los egresados de la Universidad de Guadalajara es alta debido al nivel académico del programa educativo de la carrera de médico cirujano y partero, ya que está diseñado para el desarrollo de conocimientos y habilidades que hacen que nuestros egresados sean competentes en el campo laboral, lo cual se refleja en el número de estudiantes que logran acceder a una especialidad médica, y en mayores oportunidades de inserción en el campo laboral, ya sea público o privado.

La Universidad de Guadalajara cuenta con hospitales escuela y convenios con hospitales, tanto del sector público como privado, para la realización de ciclos clínicos, internado y servicio social, lo que permite que al momento de su egreso sean fuentes de empleo para nuestros estudiantes, tanto a nivel local como nacional, quienes ocupan puestos en las áreas médico quirúrgicas, directivas y administrativas de las áreas de la salud.

Por otra parte, nuestros egresados se insertan en actividades laborales que incluyen docencia e investigación, y se desempeñan en cargos como profesores, directores, rectores, etcétera.

Se han realizado acciones como la reciente modificación al plan de estudios alineado a las tendencias internacionales en la formación médica, lo cual permitirá egresar médicos más capaces y preparados para la competencia en el campo laboral, no solamente a nivel nacional, sino internacional. 


\section{Debilidades}

No existe un plan estratégico objetivo, concreto, medible y con acciones que fortalezcan la inserción laboral de los egresados del PA.

Falta un programa de seguimiento de egresados adecuado para el PA que nos permita identificar la inserción de todos nuestros egresados a nivel local, nacional e internacional y que permita retroalimentar el PA en este rubro.

\section{Plan de mejoramiento}

El plan estratégico objetivo, concreto, medible y con acciones fortalece la inserción laboral de los egresados del PA y permite identificar estrategias y tomar acciones para fortalecer la inserción laboral de nuestros egresados a nivel local, nacional e internacional.

El programa de seguimiento de egresados adecuado para el PA permite identificar la inserción de todos nuestros egresados a nivel local, nacional e internacional.

Calificación: 9-Alta

\subsubsection{Percepción de los estudiantes, profesores y personal administrativo}

Fortalezas. Los resultados de la encuesta que se aplica al egreso de los estudiantes muestran una percepción favorable de los egresados en relación con la calidad y pertinencia del programa educativo en el contexto local.

En lo relacionado con el contexto internacional, son pocos los estudiantes que realizan estancias académicas o profesionales en el extranjero; sin embargo, en los pocos que lo han hecho se tiene una percepción excelente del desempeño del egresado.

Una evidencia de percepción adecuada sobre la calidad del PE es la participación de alumnos de diversos estados del país que presentan su solicitud para cursar la carrera de médico, cirujano y partero en el CUSur; en varios de estos casos, los aspirantes ingresan y cursan la carrera de manera satisfactoria.

Debilidades. No se aplican mecanismos suficientes de evaluación que midan la percepción y pertinencia del PE a partir de la opinión que emitan los estudiantes y profesores en el ámbito local, nacional o internacional.

Plan de mejoramiento. Se desarrolla un plan estratégico que permite medir permanentemente la opinión sobre la calidad y pertinencia del programa académico a partir 
de la opinión que emite el $70 \%$ de los alumnos, profesores, directivos y administrativos a nivel local, nacional e internacional.

Calificación: 8-Medianamente alta.

\subsubsection{Egresados inscritos en el posgrado}

Fortalezas. La gran mayoría de los egresados del Centro Universitario del Sur aspira a realizar estudios de posgrado al término de la licenciatura, por ejemplo, una especialización en las ramas médicas y quirúrgicas, por lo que se inscriben al Examen Nacional de Residencias Médicas (ENARM), ya que al ser aceptados ingresan a los hospitales del Sistema Nacional de Salud como becarios cuyos ingresos les permiten el sustento durante su preparación y, al término de su posgrado, ser contratados por estas instituciones o realizar su ejercicio en el sector privado.

Se tiene un análisis comparativo con las universidades nacionales de los últimos 14 años, en el cual se muestra que los alumnos egresados del Centro Universitario del Sur que han realizado el examen ENARM han estado dentro de los primeros 30 lugares de 94 universidades participantes en 2014, con un promedio de 62.56 puntos.

En lo que respecta al posgrado, a través del ENARM es admitido $31.56 \%$ de los aspirantes en promedio en los últimos 14 años.

Otro porcentaje de alumnos realiza posgrados de maestrías y doctorados en la Universidad de Guadalajara, instituciones educativas particulares, así como en universidades de otros países.

Se cuenta con alumnos egresados con actividades relevantes en el posgrado, así como actividades de investigación relevantes y publicaciones de investigación.

Debilidades. No se cuenta con mecanismos sistematizados que permitan conocer los resultados cuantitativos y cualitativos de ingreso y egreso del posgrado, así como de investigación o publicaciones de los egresados que cursan posgrados.

No se cuenta con patentes registradas o la participación en coautoría o derecho de autor de estudiantes de posgrado a nivel de los egresados.

Plan de mejoramiento. Se han implementado mecanismos sistematizados que permiten conocer los resultados cuantitativos y cualitativos de ingreso y egreso a nivel de posgrado, 
así como en el área de investigación y publicaciones de los egresados que cursan posgrados.

Se desarrolla una política institucional de apoyo a la creación de patentes, así como de motivación para la participación en coautoría o derecho de autor de estudiantes de posgrado.

Calificación: 8-Medianamente alta.

\subsubsection{Participación de egresados en la mejora de la formación}

Fortalezas. Los egresados del programa educativo de médico cirujano y partero de la Universidad de Guadalajara tienen una inserción constante a los colegios y asociaciones médicas; gran parte de los especialistas egresados de nuestro programa educativo tiene una participación activa como miembros de colegios de profesionales, desempeñando cargos directivos en estas instancias a nivel local y nacional, los cuales son un referente para la consulta en el ámbito académico y de la salud.

Gran parte de los egresados que participa en los colegios; se desempeñan también como profesores de la Universidad de Guadalajara y son miembros del Comité Consultivo Curricular, el cual desarrolla estrategias para la actualización del programa de estudio de médico cirujano y partero, siendo entonces los egresados los que, de acuerdo con su experiencia profesional, docente y grados académicos (especialidad, maestría y doctorado), generan las propuestas para la mejora del programa.

Por otra parte, en la Universidad de Guadalajara se cuenta con diversos institutos y centros de investigación en diferentes disciplinas de la salud, donde existe gran participación de nuestros egresados con un nivel académico ejemplar, Dicha participación se promueve desde el nivel de pregrado con la incorporación temprana a la investigación para que, posteriormente, participen en este tipo de actividades una vez que egresen.

Debilidades. No se cuenta con un adecuado programa de seguimiento de egresados en la red universitaria que permita de manera más precisa identificar la participación de nuestros egresados en colegios profesionales.

\section{Plan de mejoramiento}


Generar un programa adecuado de seguimiento de egresados que permita identificar la participación de todos nuestros egresados en colegios profesionales.

Calificación: 8-Medianamente alta.

\subsubsection{Gráfica del nivel en la calidad del programa académico evaluado}

-La gráfica 1 muestra que la apreciación de los evaluadores externos es considerada como el impacto social del programa académico evaluado. El círculo interno del color rojo es considerado como nivel incipiente de calidad, la línea amarilla como calidad media y la verde como calidad alta.

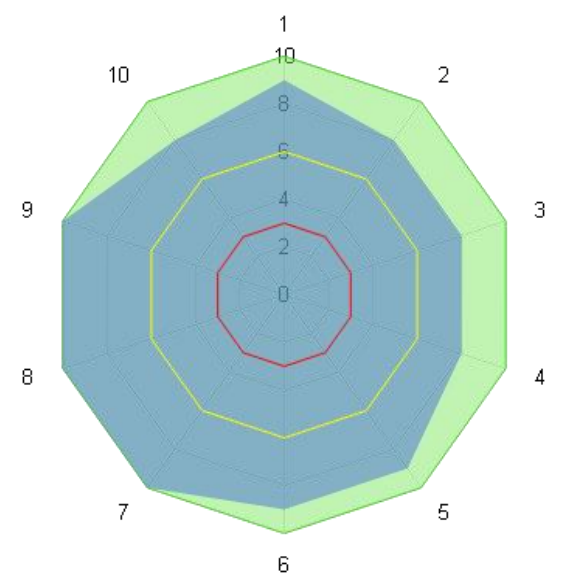

Gráfica 1.

1. Coherencia entre la visión, la misión y los objetivos planteados en la génesis de la formación con los resultados actuales, 2. Participación de entes internos y externos a la institución en la planeación, 3. Pertinencia y competitividad en el ámbito nacional e internacional, 4. Reconocimiento de la sociedad a los egresados por su desempeño, 5. Competitividad de los egresados ante similares externos, 6. Percepción de la comunidad científica, colegios especializados, egresados y empleadores, 7. Impacto en la inserción laboral nacional e internacional, 8. Percepción de los estudiantes, profesores y personal 
administrativo, 9. Egresados inscritos en el posgrado, 10. Participación de egresados en la mejora de la formación.

\subsubsection{Análisis externo del CEE del programa académico (PA) evaluado}

\subsubsection{Coherencia entre la visión, la misión y los objetivos planteados en la génesis de la formación con los resultados actuales}

\section{Fortalezas}

La institución tiene una misión y una visión claramente formuladas; estas corresponden a la naturaleza de la institución y son de dominio público. Dicha misión se expresa en los objetivos, en los procesos académicos y administrativos y en los logros de cada programa. En ella se explica el compromiso institucional con la calidad y con referentes universales de la educación superior.

Además, refleja la formación integral de los estudiantes como personas conscientes, competentes profesionalmente, íntegros moralmente y proyectados hacia el continuo mejoramiento humano, profesional y social, bajo el auspicio de una óptima preparación científica y tecnológica. Es evidente la vinculación y la ilación entre el Plan de Desarrollo Institucional 2014-2030 (PDI 2014-2030) y el Plan de Desarrollo del Centro Universitario de Ciencias de la Salud 2014-2030 (PDCUCS 2014-2030), que marcan las pautas para el desarrollo de los programas académicos (PA) con el soporte del Plan de Desarrollo de los Programas (PDP), entre los que se encuentra la licenciatura de médico cirujano y partero (LMCP). Sin duda, es una verdadera fortaleza que el $\mathrm{PDP}^{12}$ se modifique y adapte cada vez que se actualiza el PDI y que, además, se haga tomando en consideración las experiencias acumuladas en el periodo trascurrido. De esta forma, se mantiene la coherencia entre los diversos elementos contemplados en ambos planes, y con un desarrollo ascendente en calidad y pertinencia.

\section{Debilidades}

Durante las visitas al Centro Universitario, y en entrevistas a los evaluadores internos (EI), estos refirieron que entre los sectores de la sociedad que han participado en la elaboración 
del PDP del PA de la LMCP de la Universidad de Guadalajara, se encuentran los docentes, egresados, empleadores, colegios profesionales, administrativos, alumnos y usuarios; sin embargo, plantean que hasta ahora no ha sido tan activa la participación de los sectores de la sociedad en la elaboración del PDP. En las entrevistas se les preguntó cuáles sectores no participaron, y refirieron que era una apercepción subjetiva, y que consideraban que debería participar un mayor número de personas del sector social, sin mencionar quiénes más.

\section{Plan de mejoramiento}

Se coincide con los evaluadores internos (EI), ya que la Universidad de Guadalajara tiene claramente definidos en el Plan de Desarrollo Institucional (PDI) 2014-2030 y en el Plan de Desarrollo del CUSur 2014-2030 los objetivos y estrategias con indicadores para dirigir los esfuerzos y recursos, y de esta manera dar cuenta de los avances o retrocesos para el cumplimiento de las metas proyectadas, sobre todo aquellas relacionadas con el eje temático de la docencia y el aprendizaje.

Por otro lado, se recomienda una mayor (en cantidad) participación de los distintos sectores de la sociedad vinculados al área de la salud, como muy bien lo plantean los evaluadores internos (EI), ya que el PA de la LMCP es el de mayor demanda en la red universitaria.

Calificación: 10-Muy alta o excelente

\subsubsection{Participación de entes internos y externos a la institución en la planeación}

\section{Fortalezas}

En la Universidad de Guadalajara los planes PDI y PDCU, que dan funcionalidad a la red universitaria e inciden sobre los PA de la LMCP, están encauzados al desarrollo permanente de las acciones relativas a la planeación, programación, asignación de presupuesto y evaluación, cimentadas en un sistema formal institucional en permanente observación. En el proceso de actualización del PDI 2014-2030 se evidenció una amplia participación de la comunidad universitaria y la sociedad, que se llevó a cabo a través de una metodología de carácter abierto, situación que fue corroborada durante las visitas al CUCS.

En tal sentido, refirieron los EI que la institución contempla en su PDI $^{13}$ la capacitación de su personal en aras de una gestión pertinente, que les permita contribuir al cumplimiento de 
los objetivos y metas institucionales, en cuanto a fortalecer el diseño del plan de gestión de los programas educativos.

Debilidades. Plantearon los evaluadores internos (EI) durante las entrevistas, la poca internacionalización de los egresados; sin embargo, es importante iniciar con la consolidación a nivel internacional del docente y del estudiante del PA de la LMCP de la red de medicina.

Plan de mejoramiento. Plantearon los EI una revisión del diseño curricular de la LMCP cada 10 años, situación en la que coincidimos, considerando, además, que el plan de mejoramiento debe estar encaminado fundamentalmente a incrementar la participación de expertos externos en la planificación y evaluación de programas de pregrado, como es el caso de la LMCP.

Calificación: 9-Alta

\subsubsection{Pertinencia y competitividad en el ámbito nacional e internacional}

Fortalezas. El programa de la LMCP de la Universidad de Guadalajara tiene una fuerte presencia en el estado de Jalisco, debido a que se imparte en un centro temático y en cuatro centros regionales, permitiendo el ingreso de estudiantes no solo de origen regional, sino también nacional. El programa de la LMCP ha sido reconocido por la Secretaría de Educación Pública (SEP) de México como un programa de calidad. El egresado puede obtener el reconocimiento de sus estudios por la SEP al realizar el examen de certificación de EGEL-CENEVAL ${ }^{10}$, de acuerdo con el marco jurídico de México. La Universidad de Guadalajara, a través del programa académico de la LMCP en el CUCS, permite que la ubiquen entre las 9 universidades que pertenecen al Padrón de Programas de Licenciatura de Alto Rendimiento Académico-EGEL. La publicación de escalas universitarias se ha constituido en un referente importante en la valoración social del desempeño de las instituciones de educación superior. El Examen Nacional para Aspirantes a Residencias Médicas $(E N A R M)^{11}$ es un instrumento de medición de conocimientos de medicina general. La tendencia del ingreso de sustentantes de la Universidad de Guadalajara es ligeramente a la alza a través de los años (2009-2014), y ocupa segundo lugar en promedio y número de admitidos en ENARM 2014, si consideramos más de 1000 sustentantes, 
colocando a la Universidad de Guadalajara en una muy adecuada posición a nivel nacional. Es importante acotar que tanto en el 2014 como en el 2015 el promedio más alto a nivel nacional lo obtuvo un egresado del PA de la LMCP de la Universidad de Guadalajara. El programa de la $\mathrm{LMCP}^{14}$ es relevante académicamente y responde a necesidades locales, regionales, nacionales e internacionales. El programa ha definido un proyecto educativo coherente con el proyecto institucional, en el cual se señalan los objetivos, los lineamientos básicos del currículo, las metas de desarrollo, las políticas y estrategias de planeación y evaluación y el sistema de aseguramiento de la calidad. El programa es de dominio público. Toda la información anteriormente referida fue corroborada durante las visitas y entrevistas realizadas.

Por otro lado, es importante acotar que México ha ratificado y se ha adherido a la denominada Apostilla de La Haya (un convenio para la eliminación del requisito de "Legalización para documentos públicos extranjeros o convenio de La Haya") ${ }^{15}$ que reconoce la eficacia jurídica de un documento público emitido en otro país firmante de dicho convenio, entre los que se encuentran los títulos emitidos por entes gubernamentales o privados, como resulta ser la titulación de la LMCP. El trámite de apostillaje consiste en colocar sobre el propio documento público una apostilla que certificará internacionalmente la autenticidad de la firma de los documentos públicos expedidos en cada país miembro, reconociendo por consiguiente la autenticidad de los documentos que se han expedido en otros países y llevan la apostilla, ${ }^{14}$ lo que permite reconocer la formación obtenida en un área de conocimiento y, por ende, ejercer profesionalmente a un individuo en otro país signatario.

Debilidades. Los EI hicieron referencia al poco crecimiento de la movilidad a nivel internacional de estudiantes y académicos y a la necesidad de expansión de los cuerpos académicos de investigación en el ámbito internacional. Sin embargo, durante la visita y las entrevistas a estudiantes y profesores se pudo conocer que, en el presente año, más de 120 estudiantes extranjeros realizaron actividad académica en el PA de la LMCP de la red de medicina, y un menor número de estudiantes de la Universidad de Guadalajara realizó movilidad internacional y posteriormente solicitó la convalidación de las actividades realizadas en las distintas sedes académicas internacionales. 
Plan de mejoramiento. Las debilidades pueden ser superadas a través de los objetivos planteados en la internacionalización del PDI 2014-2030, que considera la consolidación de la internacionalización en las funciones sustantivas de la Universidad de Guadalajara. Además, se recomienda mantener e incrementar la movilidad internacional de estudiantes y académicos, como muy bien lo plantea el PDI.

Calificación: 10-Muy alta o excelente.

\subsubsection{Reconocimiento de la sociedad a los egresados por su desempeño}

Fortalezas. Es un Centro con méritos reconocidos a través de evaluaciones con criterios de calidad en sus egresados, ubicados en los primeros 30 lugares en el ENARM, y en el lugar 27 en el año 2014. Alto rendimiento de calificación en la evaluación del CENEVAL por altas calificaciones de los egresados en los dos últimos años. Dos acreedores a premio 2014 y premiados y más de 60 estudiantes con un rendimiento satisfactorio. Premios de excelencia EGEL y Pfizer en uno de sus egresados; premios en congresos.

Debilidades. No hay estudios sobre el impacto de sus egresados en la sociedad e instituciones donde desarrollan su labor luego de egresados.

Plan de mejoramiento. Un plan estratégico, con metas a corto y mediano plazo, sería plausible para evaluar este ítem, donde se haga un seguimiento de sus egresados no solo en el campo laboral, sino en el área de investigación.

Calificación: 10 -Muy alta o excelente.

\subsubsection{Competitividad de los egresados ante similares externos}

Fortalezas. Como indicador de calidad, la acreditación nacional este año y los tres años anteriores hace ver a sus egresados con competencias para el desempeño. Otro criterio de calidad es el hecho de que pertenece al Padrón de Alto Rendimiento del CENEVAL, por sus altas calificaciones en los egresados en los últimos dos años. En el 2015, de 188 sustentantes que presentaron el XXXIX Examen Nacional para Aspirantes a Residencias Médicas, ${ }^{14} 60$ fueron seleccionados (31.92\%) con un promedio general de conocimientos médicos de 62,557. El número de estudiantes evaluados por EGEL se ha incrementado de 290, en el 2009, a 985 en el 2014 (339.55 \% de aumento en 5 años, más de tres veces). 
Debilidades. No cuentan con la sistematización de datos y seguimiento a los egresados, para compararlos con los del resto del país y a nivel internacional (su desempeño laboral, académico y de investigación, este último a través de sus publicaciones).

\section{Plan de mejoramiento}

Sería interesante sistematizar la data para el seguimiento de los egresados. Se propone un plan estratégico que permita el seguimiento de los egresados y su desempeño laboral, académico y de investigación, este último a través de sus publicaciones.

Calificación: 10-Muy alta o excelente.

\subsubsection{Percepción de la comunidad científica, colegios especializados, egresados y empleadores}

Fortalezas. Los egresados del programa de la LMCP de la red universitaria de la Universidad de Guadalajara son reconocidos por la calidad de la formación que reciben, y se destacan por su desempeño en la disciplina, condición que se evidenció en la percepción de los empleadores, la cual fue corroborada por los egresados durante las visitas y entrevistas realizadas. Además, es importante referirnos nuevamente al ENARM, donde los egresados de la Universidad ocuparon segundo lugar en promedio y número de admitidos en el 2014, si consideramos más de 1000 sustentantes, lo que representa un reconocimiento a nivel nacional. Es importante acotar que se mantiene un vínculo interesante entre los egresados y la institución académica, que se encuentra reflejado en el marco jurídico de la misma.

Debilidades. Coincidimos con las referidas por los EI, de realizar seguimiento a los egresados de manera de conocer sus logros, además de establecer convenios nacionales que faciliten la inserción de un número determinado de egresados al campo laboral.

Plan de mejoramiento. Consideramos que las estrategias de los EI son adecuadas para superar las debilidades, entre las que se encuentra el contar con un programa de seguimiento de los egresados más sistemático, mantener un contacto más directo con los empleadores y colegios de profesionales, etcétera.

Calificación: 9-Alta 


\subsubsection{Impacto en la inserción laboral nacional e internacional}

Fortalezas. En investigación reciente realizada por la Universidad de Guadalajara a empleadores del ámbito de las instituciones de salud pública y privada, se obtuvo como resultado que el $100 \%$ opinó favorablemente de la calidad y pertinencia que presenta el programa educativo en la LMCP; igual porcentaje consideró favorablemente el desempeño laboral observado en los egresados de la LMCP, y consideró como muy importante que los egresados cuenten con capacidad para identificar y solucionar problemas, conocimientos técnicos de la disciplina, ética, valores, buena relación médico-paciente y capacidad para realizar procedimientos médicos. Lo anteriormente referido sustenta el hecho de por qué la inserción al campo laboral por parte de los egresados de la Universidad de Guadalajara en la LMCP es alta. ${ }^{19}$ Además, el egresado de esta licenciatura logra acceder a realizar una especialidad médica en un porcentaje importante, lo cual les abre aún más la oportunidad de inserción en espacios laborales, ya sean públicos o privados. Es importante acotar que todo lo anteriormente referido fue corroborado durante las visitas y entrevistas. Otro campo de trabajo al cual tiene acceso el egresado de la LCMP es el académico, que incluyen docencia e investigación.

Debilidades. Se comparten las debilidades, referidas por los EI, de que no existe un plan estratégico ponderable y con acciones que fortalezcan la inserción laboral de los egresados del PA de la LMCP de la red de medicina. Adicionalmente, ausencia de seguimiento sistematizado de los egresados del PA de la LMCP que permita identificar el nivel de inserción de los mismos al campo laboral a nivel local, nacional e internacional; es decir, falta un sistema informático de retroalimentación con los egresados.

Plan de mejoramiento. Concordamos con los planteamientos de los EI, ratificados durante las entrevistas, sobre la necesidad de desarrollar un plan estratégico concreto, ponderable y con acciones para fortalecer la inserción laboral de los egresados del PA de la LMCP de la red de medicina, además de identificar estrategias y tomar acciones para fortalecer la inserción laboral y el seguimiento de los egresados a nivel local, nacional e internacional. Calificación: 9-Alta.

\subsubsection{Percepción de los estudiantes, profesores y personal administrativo}


Fortalezas. Consideramos que la aplicación de encuestas a los egresados es una fortaleza, ya que permite evaluar cualitativamente la percepción que manejan de la institución, en cuanto a calidad y pertinencia de la licenciatura. El instrumento está bien estructurado y es de fácil manejo y comprensión. En las entrevistas a estudiantes, docentes y egresados se pudo corroborar el sentido de pertenencia que tienen hacia su máxima casa de estudios. Al personal administrativo se le notó la mística en el desempeño del trabajo.

Debilidades. Aunque es un instrumento para que los alumnos evalúen a sus profesores, se aplica a pocos docentes.

Plan de mejoramiento. Mantener las evaluaciones por año lectivo, mejorando las metas propuestas. Crear y aplicar un instrumento dirigido a los estudiantes. Se pudo corroborar en la visita que el instrumento está en construcción.

Calificación: 9 -Alta

\subsubsection{Egresados inscritos en el posgrado}

Fortalezas. Sus egresados llevan un alto índice de calidad acreditado por el CENEVAL, además de las acreditaciones nacionales en los últimos años. En el ENARM (Examen Nacional de Residencias Médicas), que permite el ingreso a los médicos a la realización de sus especializaciones en los diferentes hospitales, la licenciatura cuenta con unos estándares de calidad para ingresar a la carrera, un programa completo en todas sus dimensiones, que los hace competitivos, aunque un alto porcentaje ingresa- como becarios, con las ventajas que esto representa. Además, se tiene registro en el ENARM de los últimos 14 años, con un promedio del porcentaje de admisión de 31.56\%, donde CUSur ocupó el lugar 32 de 94 universidades participantes en el año 2014. Existe un registro de tesis publicadas 20102014.

Debilidades. No presentan un seguimiento interno de los egresados que se encuentran realizando posgrados ni evaluaciones cualitativas y cuantitativas luego de culminadas sus especialidades.

Plan de mejoramiento. Minimizar las debilidades; se sugiere, si lo permite su estructura legal, crear una unidad de seguimiento, con una base de datos de los egresados, de manera que al optar y/o ingresar a los posgrados, la Universidad tenga conocimiento de ello; de 
igual manera al egresar de sus estudios superiores, como las especializaciones, maestrías y doctorados.

Calificación: 9-Alta

\subsubsection{Participación de egresados en la mejora de la formación}

Fortalezas. Los egresados del PA de la LMCP de la red de medicina son reconocidos por la calidad de la formación que reciben y se destacan en su ejercicio profesional; esto se hace evidente por el desempeño de los egresados en puestos directivos públicos y privados en las áreas de la salud, educación pre y posgrado y a nivel de colegios profesionales. Cabe resaltar que algunos forman parte del personal docente de la Universidad de Guadalajara. Algunos de los egresados mostraron un notable desempeño en el ámbito de la salud a nivel internacional, condición que fue confirmada durante las visitas y entrevistas.

Debilidades. Al igual que en ítems anteriores, coincidimos con las debilidades referidas por los EI durante sus apreciaciones en la evaluación interna y mientras se realizaron las entrevistas, cuando plantean de manera general que existe deficiencia en el seguimiento de los egresados por no contar con una herramienta informática interactiva, pues, de lo contrario, se lograría un control del destino laboral y académico de cada uno de ellos; además, de no brindar la posibilidad de que los egresados participen en los procesos de mejoramiento de los PA de la LMCP a través de foros a distancia.

Plan de mejoramiento. Consideramos que las estrategias de los EI son adecuadas para superar las debilidades, cuando plantean generar un programa de seguimiento de los egresados.

Calificación: 9-Alta

\subsubsection{Gráfica de rubro}

En la gráfica 2 se observa el resultado de la evaluación externa del impacto social de la formación. 


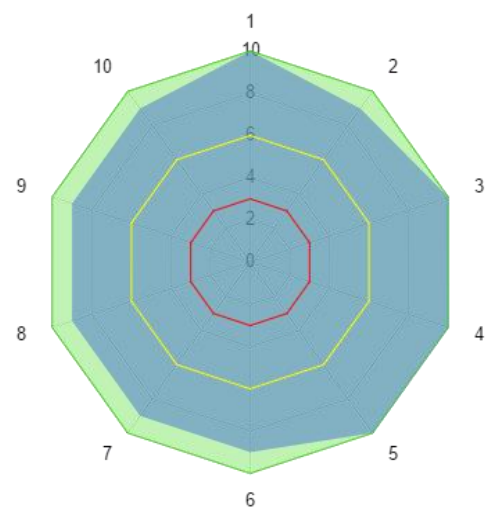

\section{Gráfica 2}

1. Coherencia entre la visión, la misión y los objetivos planteados en la génesis de la formación con los resultados actuales, 2. Participación de entes internos y externos a la institución en la planeación, 3. Pertinencia y competitividad en el ámbito nacional e internacional, 4. Reconocimiento de la sociedad a los egresados por su desempeño, 5. Competitividad de los egresados ante similares externos, 6. Percepción de la comunidad científica, colegios especializados, egresados y empleadores, 7. Impacto en la inserción laboral nacional e internacional, 8. Percepción de los estudiantes, profesores y personal administrativo, 9. Egresados inscritos en el posgrado, 10. Participación de egresados en la mejora de la formación.

\section{Discusiones}

La institución tiene una misión y una visión claramente formuladas; estas corresponden a la naturaleza de la institución y son de dominio público. Dicha misión se expresa en los objetivos, en los procesos académicos y administrativos y en los logros de cada programa. En ella se explica el compromiso institucional con la calidad y con referentes universales de la educación superior. Además, refleja la formación integral de los estudiantes como personas conscientes, competentes profesionalmente, íntegros moralmente y proyectados hacia al continuo mejoramiento humano, profesional y social, bajo el auspicio de una óptima preparación científica y tecnológica. 
Es evidente la vinculación y la ilación entre el Plan de Desarrollo Institucional 2014-2030 (PDI 2014-2030) y el Plan de Desarrollo del Centro Universitario del Sur 2014-2030 (PDCUCS 2014-2030), que marcan las pautas para el desarrollo del programa académico (PA) con el soporte del Plan de Desarrollo de los Programas (PDP), entre los que se encuentra la licenciatura de médico cirujano y partero (LMCP). Sin duda, es una verdadera fortaleza que el PDP se modifique y adapte cada vez que se actualiza el PDI y que, además, se haga tomando en consideración las experiencias acumuladas en el periodo trascurrido. De esta forma se mantiene la coherencia entre los diversos elementos contemplados en ambos planes, con un desarrollo ascendente en calidad y pertinencia. En la Universidad de Guadalajara los planes de desarrollo que dan funcionalidad a la red universitaria e inciden sobre el PA de la LMCP están encauzados al desarrollo permanente de las acciones relativas a la planeación, programación, asignación de presupuesto y evaluación, cimentadas en un sistema formal institucional en permanente observación. En el proceso de actualización del PDI 2014-2030 se evidenció una amplia participación de la comunidad universitaria y la sociedad, que se llevó a cabo a través de una metodología de carácter abierto, situación que fue corroborada durante las visitas al CUCS. En tal sentido, refirieron los EI que la institución contempla en su PDI ${ }^{12,13}$ capacitación de su personal en aras de una gestión pertinente, que les permita contribuir al cumplimiento de los objetivos y metas institucionales, en cuanto a fortalecer el diseño del plan de gestión de los programas educativos. El programa de la LMCP de la Universidad de Guadalajara tiene una fuerte presencia en el estado de Jalisco, debido a que se imparte en un centro temático y en cuatro centros regionales, permitiendo el ingreso de estudiantes no solo de origen regional, sino también nacional. El programa de la LMCP ha sido reconocido por la Secretaría de Educación Pública (SEP) de México como un programa de calidad. El egresado puede obtener el reconocimiento de sus estudios por la SEP al realizar el examen de certificación de EGEL-CENEVAL ${ }^{12}$, de acuerdo con el marco jurídico de México. La Universidad de Guadalajara, a través del programa académico de la LMCP en el CUSur, permite que la ubiquen entre las nueve universidades que pertenecen al Padrón de Programas de Licenciatura de Alto Rendimiento Académico-EGEL.

La publicación de las escalas universitarias se ha constituido en un referente importante en la valoración social del desempeño de las instituciones de educación superior. El Examen 
Nacional para Aspirantes a Residencias Médicas (ENARM) ${ }^{14}$ es un instrumento de medición de conocimientos de medicina general. La tendencia del ingreso de sustentantes de la Universidad de Guadalajara es ligeramente a la alza a través de los años (2009-2014) y ocupa segundo lugar en promedio y número de admitidos en el ENARM 2014, si consideramos más de 1000 sustentantes, colocando a la Universidad de Guadalajara en una muy adecuada posición a nivel nacional. Es importante acotar que tanto en el 2014 como en el 2015 el promedio más alto a nivel nacional lo obtuvo un egresado del PA de LMCP. El programa de la LMCP es relevante académicamente y responde a necesidades locales, regionales, nacionales e internacionales. El programa ha definido un proyecto educativo coherente con el proyecto institucional, en el cual se señalan los objetivos, los lineamientos básicos del currículo, las metas de desarrollo, las políticas y estrategias de planeación y evaluación y el sistema de aseguramiento de la calidad. El programa es de dominio público. Toda la información anteriormente referida fue corroborada durante las visitas y entrevistas realizadas.

Por otro lado, es importante acotar que México ha ratificado y se ha adherido a la denominada Apostilla de La Haya $^{13}$ (un convenio para la eliminación del requisito de “Legalización para documentos públicos extranjeros o convenio de La Haya”), que reconoce la eficacia jurídica de un documento público emitido en otro país firmante de dicho convenio, entre lo que se encuentra los títulos emitidos por entes gubernamentales o privados, como resulta ser la titulación de la LMCP.

El trámite de apostilla consiste en colocar, sobre el propio documento público, una apostilla que certificará internacionalmente la autenticidad de la firma de los documentos públicos expedidos en cada país miembro, reconociendo por consiguiente la autenticidad de los documentos que se han expedido en otros países y llevan la apostilla, lo que permite reconocer la formación obtenida en un área de conocimiento $\mathrm{y}$, por ende, ejercer profesionalmente a un individuo en otro país signatario. En la gráfica 3 se observan las gráficas de la evaluación interna versus la evaluación externa sobre el impacto social de la formación. 

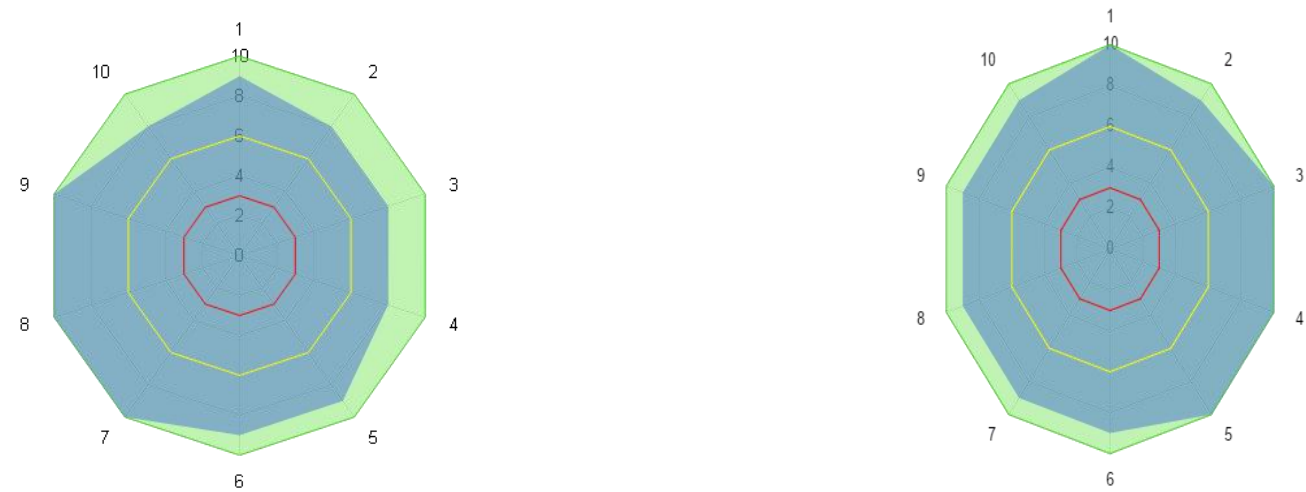

\section{Gráfica 3. Evaluación interna versus evaluación externa.}

1. Coherencia entre la visión, la misión y los objetivos planteados en la génesis de la formación con los resultados actuales; 2. Participación de entes internos y externos a la institución en la planeación; 3. Pertinencia y competitividad en el ámbito nacional e internacional; 4. Reconocimiento de la sociedad a los egresados por su desempeño; 5. Competitividad de los egresados ante similares externos; 6. Percepción de la comunidad científica, colegios especializados, egresados y empleadores; 7. Impacto en la inserción laboral nacional e internacional; 8. Percepción de los estudiantes, profesores y personal administrativo; 9. Egresados inscritos en el posgrado; 10. Participación de egresados en la mejora de la formación.

\section{Conclusiones}

\subsection{Etapa de visita y entrevistas al Centro Universitario del Sur (CUSur)}

Como parte del proceso de gestión para evaluar la calidad de la LMCP en red de la Universidad de Guadalajara, México, se ha observado durante el proceso de evaluación externa presencial en el periodo comprendido entre el 26 de noviembre de 2015 y el 27 de noviembre de 2015 lo siguiente: los comités de evaluación interna realizaron un intenso trabajo de recopilación de información, siendo muy positiva su disposición para el buen desempeño del proceso de evaluación externa del que forma parte el presente informe. Cada vez que los evaluadores externos solicitaron diferentes tipos de información, esta fue proporcionada de forma inmediata, abierta y transparente, pudiendo en la visita realizada a 
la Universidad de Guadalajara interactuar con todas las áreas administrativas y académicas. Además, un elevado compromiso institucional por desarrollar mejoras sustantivas, en lo que respecta a su oferta académica, con una gran disposición a someterse a procesos de evaluación externa para la mejora de sus programas educativos. Elevado entusiasmo y compromiso por parte de todos los componentes de la comunidad universitaria por los procesos de mejoras y calidad. Asimismo, durante las visitas realizadas y las entrevistas mantenidas en el proceso de evaluación con las distintas instancias académicoadministrativas, y con el propio comité de evaluación interna, se evidenciaron los puntos antes señalados.

\subsection{Sobre el dictamen}

Posterior al análisis de la evaluación interna, de la apertura del proceso institucional, de la documentación presentada, las visitas realizadas, las entrevistas mantenidas en el proceso de evaluación con las distintas instancias académico-administrativas y con el propio comité de evaluación interna, evidenciaron los puntos señalados a continuación, con los cuales este CEE llegó al siguiente dictamen, según cada uno de los rubros evaluados: existe un gran compromiso por parte de la institución con la excelencia, lo cual incluye al rector, al personal docente y al personal administrativo y de apoyo. También un sentido de pertenencia de los estudiantes con la Universidad y el PA. Hay una voluntad institucional para llevar adelante el programa, consolidarlo y procurar su internacionalización. La visita se enmarcó en una actitud de apertura, generosidad y transparencia que estuvo presente en cada una de las actividades, y gracias a ello se cumplió a cabalidad la labor de diagnóstico y diálogo por parte del CEE con la comunidad del PA. Lo anterior es reflejo del compromiso institucional con la calidad. La Universidad de Guadalajara cuenta con un amplio y completo marco normativo que se circunscribe a reglamentar de manera eficaz y pertinente las diversas funciones sustantivas de la institución. La institución tiene una misión y una visión claramente formuladas; estas corresponden a la naturaleza de la institución y son de dominio público. Dicha misión se expresa en los objetivos, en los procesos académicos y administrativos y en los logros de cada programa; en ella se explicita el compromiso institucional con la calidad y con los referentes universales de la educación superior. Se observó coherencia entre la misión y la visión institucional y el PA de la LMCP del CUSur, 
que responde a necesidades del contexto, locales, regionales, nacionales e internacionales. Además, refleja la formación integral de los estudiantes como personas conscientes, competentes profesionalmente, íntegros moralmente y proyectados hacia el continuo mejoramiento humano, profesional y social, bajo el auspicio de una óptima preparación científica y tecnológica. La Universidad de Guadalajara aplica mecanismos universales y equitativos de ingreso de estudiantes, que son conocidos por los aspirantes y se basan en la selección por méritos y capacidades intelectuales, sustentadas en un marco jurídico. A nivel institucional se denota un compromiso y unas estrategias congruentes con la identidad institucional, para favorecer la internacionalización desde un eje temático del Plan de Desarrollo Institucional 2014-2030. El eje contempla la transformación curricular para formar ciudadanos globales y profesionales con capacidad para desempeñarse a nivel internacional; impulsar, mediante la movilidad internacional, el desarrollo de una masa crítica que contribuya a mejorar la endogamia académica; fortalecer la investigación y la generación de recursos externos de cooperación; desarrollar acuerdos de colaboración para programas compartidos y atraer talento internacional de alto nivel. Por otro lado, la vinculación entre el Plan de Desarrollo Institucional 2014-2030 (PDI 2014-2030) y el Plan de Desarrollo del Centro Universitario de Ciencias de la Salud 2014-2030 (PDCUCS 20142030) marca las pautas para el desarrollo de los programas académicos (PA) con el soporte del Plan de Desarrollo de los Programas (PDP), entre los que se encuentra la licenciatura de médico cirujano y partero. La Universidad de Guadalajara cuenta con una infraestructura y medios materiales muy adecuados, y es importante resaltar el esfuerzo en la inversión realizada en los últimos años. El programa aplica las políticas de ingreso y permanencia establecidas a nivel institucional, y logra eficazmente la culminación del alumnado en los tiempos previstos, ya que es flexible a las necesidades del estudiante. Las asesorías y tutorías que ofrece el programa se reflejan en los niveles bajos de deserción que se registran en el programa; no obstante, pareciera determinante el hecho de que debería existir una planta profesoral con mayor tiempo de contratación para cubrirlas, cuando se observa que la matrícula va en ascenso. La evolución en el desarrollo de la investigación es evidente y se manifiesta en los resultados vinculados a la formación, la participación de estudiantes en actividades académicas nacionales e internacionales y las publicaciones de profesores en revistas reconocidas a nivel nacional e internacional, así como libros de interés para los 
estudiantes y el público en general. La producción de investigación realizada por los académicos asociados al programa académico es significativa, y les es reconocido por el Sistema Nacional de Investigadores (SNI). La institución cuenta con una planta profesoral de alta calidad, comprometida con el PA y el desarrollo de las funciones sustantivas. El programa registra una dinámica importante de participación de sus profesores como asistentes y ponentes en eventos internacionales organizados por instituciones de prestigio, en estancias de investigación y en cursos de actualización pedagógica y disciplinaria. Se reconoció que la producción de investigación realizada por los académicos asociados al programa académico es significativa y les es reconocido por el (SNI). Se observó que los comités de evaluación interna realizaron un intenso trabajo de recopilación de información y fue muy positiva su disposición para el buen desempeño del proceso de evaluación externa. Cada vez que se solicitaron diferentes tipos de información, esta fue proporcionada de forma inmediata, abierta y transparente, permitiendo en la visita realizada al centro universitario interactuar con todas las áreas administrativas y académicas. El programa acoge el modelo académico institucional con una estructura semiflexible del plan de estudios, el cual se organiza en unidades de aprendizaje que derivan en el establecimiento de competencias profesionales. En virtud de la implementación del nuevo plan de estudios que está incorporado a partir del calendario 2014-B, se denota una congruencia y pertinencia en el diseño curricular del plan de estudios con los objetivos, la organización de las materias y sus contenidos, la transición progresiva y agrupación de estos, por ejes disciplinares, y las actividades de autogestión asociadas en el plan de estudios. Los planes de estudio de la licenciatura contemplan unidades de aprendizaje de formación básica común y particular obligatorias, de formación especializante selectiva con orientaciones médica, quirúrgica, en investigación y en atención a la familia, y un área de formación optativa abierta. La realización de prácticas profesionales en los alumnos de la carrera se lleva a cabo durante su formación, los cuales son supervisados por profesores calificados. La naturaleza práctica de la disciplina permite cumplir con el compromiso social expresado en la misión y la visión institucional. Se plantea que la alta demanda de atención de los alumnos, en especial en la práctica profesional, sugiere que la institución considere la posibilidad de un incremento en la planta profesoral para garantizar un adecuado acompañamiento. Durante el proceso formativo de los alumnos de esta licenciatura, y 
siguiendo los lineamientos académicos señalados en el plan de desarrollo de la Universidad de Guadalajara, del Centro Universitario del Sur, se desarrollan habilidades para que el egresado establezca una vinculación cercana con la sociedad; también se le brinda la oportunidad de conocer las necesidades de su entorno y las políticas de globalización para que pueda incorporarse y desarrollar sus conocimientos en beneficio de la comunidad nacional e internacional. El CUSur cuenta con una infraestructura de laboratorios, clínica de salud, espacios de simulación con tecnología de punta para la enseñanza y centros médicos externos vinculados al PA. La infraestructura física y tecnológica del Centro Universitario se considera, en términos generales, pertinente y cubre la mayoría de las necesidades básicas de formación de los estudiantes. Se reconoce el esfuerzo de la institución y del programa por modernizar la infraestructura y la dotación de apoyos académicos. La biblioteca cuenta con una amplia gama de base de datos y libros especializados, y brinda talleres y cursos en áreas que no fueron consideradas en el PA, como el dominio de otro idioma y el uso de las TIC, que son vitales en la formación e internacionalización tanto de estudiantes y egresados como de docentes. Existe prestación de servicios a la comunidad de profesores y alumnos en forma continua, tanto en actividades de educación, prevención y tratamiento en instalaciones fijas y móviles como en campañas específicas de promoción de salud y detección de la enfermedad. La Universidad de Guadalajara en general, y el Centro Universitario del Sur (CUSUR) en particular, tienen convenios firmados con varias de las instituciones de salud más importantes a nivel federal e internacional. Los estudiantes del programa han participado en eventos académicos de carácter internacional, como veranos de investigación científica y congresos internacionales que se realizan en el país. El programa registra un incremento en la movilidad académica internacional en los últimos años, y cuenta con apoyo financiero de la Unidad de Becas y la divulgación de las oportunidades por parte de la Coordinación General de Cooperación e Internacionalización. Existe una clara estructura organizacional que establece los lineamientos para satisfacer las necesidades y demandas de estudiantes, profesores, directivos y personal administrativo. Es importante acotar que, durante las distintas visitas y entrevistas previstas para la evaluación del PA de la LMCP del CUSur, se comprobó toda la información y anexos colocados por el CEI. Los egresados del programa 
son reconocidos por la calidad de la formación que reciben y se destacan por su desempeño profesional.

\subsection{En relación con las debilidades}

Lo más significativo en cuanto a las debilidades fue lo relativo a la necesidad de sistematización de la información, tal como fue la relación de los egresados y lo relativo a las actividades de extensión. Otro aspecto significativo es la necesidad de garantizar la inclusión y prosecución en la carrera a los grupos vulnerables, en consonancia con la infraestructura para esos grupos, que no se encuentra cien por ciento adecuada. La instalación de rampas para discapacitados es una prioridad. Se sugiere que se adopte un sistema de asignación y transferencia de créditos que se aplique internacionalmente, y el manejo de un segundo idioma. La baja receptividad de los estudiantes a la idea de los intercambios podría deberse a ciertos obstáculos que dificultan la movilidad de los estudiantes. Algunas de estas limitaciones estarían relacionadas directamente con el PA, pues el aprendizaje de otro idioma, mandatorio para los intercambios, no tiene carácter de obligatoriedad. Además, los costos de ciertos trámites burocráticos (como las visas), así como la inversión de tiempo para cumplir con ellos, pueden desmotivar a ciertos candidatos.

Propuestas: se recomienda un incremento en la divulgación de sus fortalezas, sobre todo en redes sociales institucionales de ámbito nacional e internacional; masificar la enseñanza de un segundo idioma como parte del plan de estudios; que las propuestas sean concretas para incentivar la movilidad estudiantil y de los profesores, la publicación en revistas y libros internacionales y arbitrados, la participación (como ponentes) en eventos internacionales y el reconocimiento internacional del PA. Tal y como se ha descrito, el PA goza de un reconocimiento regional y nacional que le ha permitido consolidar su posición de liderazgo en la LMCP de México. El PA ya ha sido evaluado muy favorablemente por organismos nacionales que certificaron la calidad del programa y diseñaron algunas herramientas para su crecimiento. Los reportes de las evaluaciones anteriores realizadas por

estos organismos han resultado en un profundo examen y en un sincero proceso de reflexión y reajuste del PA. 


\section{Bibliografía}

Reynaga, O.S, (2015). Los sistemas educativos de México y Reino Unido. British Council. 